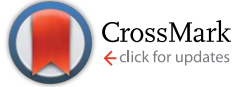

Cite this: RSC Adv., 2016, 6, 28809

\title{
The effect of block copolymer additives for a highly active polymeric metal-free oxygen reduction electrode $\uparrow$
}

\author{
Samuel R. Pering, ${ }^{\text {ab }}$ James A. Nicholas, ${ }^{\text {ab }}$ Bita Bayatsarmadi, ${ }^{c}$ Drew Evans, ${ }^{b}$ \\ Manrico Fabretto, ${ }^{b}$ Anton Blencowe, ${ }^{\text {bd }}$ Peter J. Murphy ${ }^{b}$ and Pejman Talemi*c
}

\begin{abstract}
Electrocatalytic materials play a critical role in emerging clean energy technologies such as batteries and fuel cells. Herein, we report the fabrication of a unique conducting polymer alloy thin film electrocatalyst based on poly(3,4-ethylenedioxythiophene) (PEDOT) and a polymeric additive, polyethylene glycol-polydimethylsiloxane-polyethylene glycol. These polymeric films displayed electrocatalytic activity for the Oxygen Reduction Reaction (ORR) higher than that of platinum or other polymeric electrodes. Furthermore, the metal-free polymeric electrodes were not susceptible to 'poisoning' when exposed to methanol. The fabrication parameters affecting the ORR performance of the polymeric electrodes and the mechanism of the improvement of the ORR catalysis were comprehensively investigated and benchmarked against commercial Pt electrodes. The application of the conducting polymer alloy electrode in a zinc-air battery demonstrated high power output and stable performance.
\end{abstract}

Received 6th January 2016

Accepted 11th March 2016

DOI: $10.1039 / c 6 r a 00319 b$

www.rsc.org/advances

scarcity of Pt, these catalysts exhibit degradation due to the

\section{Introduction}

Both the effects of climate change and the increasing scarcity of traditional fossil fuels have greatly increased the need to develop renewable systems for the production of energy. Solar and wind energy offer environmentally clean methods for obtaining power, although the variable nature of such energy sources necessitates the development of storage devices for sustainable energy supplies. ${ }^{1,2}$ As a result, metal-air batteries have been the subject of considerable interest due to their high theoretical capacities and energy densities. ${ }^{3,4}$ Air-electrodes are a critical component of both metal-air batteries and fuel cells, as they provide an interface for ambient oxygen to contribute to the electrochemical reaction of the battery; negating the need for refuelling and allowing the cathode to operate continuously. One of the basic requirements for a metal-air battery to achieve its theoretical capacity is an efficient catalyst for the oxygen reduction reaction (ORR). Catalysts used for oxygen reduction typically contain a noble metal, most commonly platinum (Pt), supported by a carbon scaffold. In addition to the high cost and

${ }^{a}$ Department of Chemistry, University of Bath, Claverton Down, Bath, BA2 7AY, UK ${ }^{b}$ Future Industries Institute, University of South Australia, Mawson Lakes, SA 5095, Australia

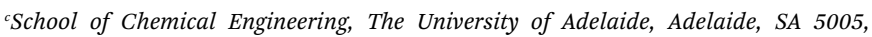
Australia.E-mail: pejman.talemi@adelaide.edu.au

${ }^{d}$ School of Pharmacy and Medical Sciences, University of South Australia, SA 5001, Australia

$\dagger$ Electronic supplementary information (ESI) available. See DOI: 10.1039/c6ra00319b instability (poisoning) of platinum. Therefore, many researchers have focussed on improving the stability and life-cycle by using additives in the support structures or by alloying the noble metals..$^{5-7}$ Atmospheric $\mathrm{CO}$ or methanol (a proposed fuel for fuel cells) can also reduce the lifetime of Pt-based air electrodes, poisoning the catalyst and reducing its overall efficiency. ${ }^{8}$ To overcome these problems transition metal compounds have been sought as Pt replacements, ${ }^{9-12}$ as well as the utilisation of novel carbon-based materials. ${ }^{4,13-16}$ In recent years, conductive polymers have started to attract significant attention as potential electrocatalytic materials. ${ }^{17-22}$ Khomenko et al. demonstrated that a number of intrinsically conducting polymers (ICPs) could catalytically reduce atmospheric oxygen, ${ }^{17}$ and it was proposed that the aromatic sections of the polymers were able to lose electron density to adsorbed oxygen, thereby reducing it. ${ }^{17,23}$ Other advantages of conductive polymers are that they are lighter, cheaper and less susceptible to poisoning compared to many metal-based cathodes. This means that polymer-based cathode batteries can potentially be cheaper, and have higher energy densities and lifetimes compared to conventional batteries. Poly $(3,4-$ ethylenedioxythiophene) (PEDOT) is an ICP that has attracted significant research interest due to its high conductivity and flexibility, ${ }^{24-26}$ and has been reported to deliver similar electrocatalytic performance to Pt when prepared utilising the vacuum vapour phase polymerisation (VPP) technique. ${ }^{19,20}$ Early studies in this field, however, necessitated the use of a gold pre-layer, which was later shown to have its own electrocatalytic effect. ${ }^{27}$ Subsequently, our group eliminated the 
Table 1 Labelling, conductivity (single layer polymer deposited on glass) and the ORR conversion current (5 layers deposited on $50 \%$ wet proofed carbon paper) of PEDOT samples based on different polymer additives

\begin{tabular}{|c|c|c|c|}
\hline Sample & Polymer additive & $\begin{array}{l}\text { Conductivity of } \\
\text { films }\left(\mathrm{S} \mathrm{cm}^{-1}\right)\end{array}$ & $\begin{array}{l}\text { ORR current at }-0.9 \\
\text { V vs. Ag/AgCl, pH } 13\left(\mathrm{~mA} \mathrm{~cm}^{-2}\right)\end{array}$ \\
\hline E-PEDOT & PEG & 206 & 5.7 \\
\hline P-PEDOT & PPG & 284 & 5.6 \\
\hline EPE-PEDOT & PEG-PPG-PEG & 2003 & 7.2 \\
\hline E30P-PEDOT & PEG (30 wt $\%) /$ PPG blend & 346 & 5.7 \\
\hline S-PEDOT & PEG-PDMS-PEG & 377 & 12.3 \\
\hline S20-PEDOT & PEG-PDMS-PEG (20 wt\%)/PEG-PPG-PEG & 1284 & 9.1 \\
\hline S50-PEDOT & PEG-PDMS-PEG (50 wt\%)/PEG-PPG-PEG & 919 & 10.1 \\
\hline S80-PEDOT & PEG-PDMS-PEG (80 wt\%)/PEG-PPG-PEG & 873 & 9.3 \\
\hline ELAT & N/A & $\mathrm{N} / \mathrm{A}$ & 4.7 \\
\hline
\end{tabular}

need for a metallic under layer by producing highly conductive PEDOT electrodes using a structure-directing copolymer PEG-PPG-PEG (PEG: poly(ethylene glycol), PPG: poly(propylene glycol)). ${ }^{16,20}$ In this paper, we will investigate the effect of this polymeric additive and how it can affect the electrocatalytic properties of PEDOT electrodes. We found that by selecting the chemistry of each of the segments of the block copolymer additive it is possible to enhance the oxygen and water absorption and improve the electrocatalytic properties of the electrode. Amongst the samples discussed in this work, PEDOT electrodes prepared with assistance of a block copolymer based on polydimethylsiloxane (PEG-PDMS-PEG) demonstrated the highest electrocatalytic activity and the best performance when incorporated in a prototype zinc-air battery.

\section{Experimental section}

\section{Preparation of air cathode}

The oxidant solutions used for polymerisation utilised $1.5 \mathrm{~g}$ copolymer, either PEG-PPG-PEG (Sigma-Aldrich, $M_{\mathrm{n}}=5.8$ $\mathrm{kDa}$ ) or PEG-PDMS-PEG (DBE-U12 and DBE-U22, Gelest) or a combination of them (Table 1), with $2 \mathrm{~g}$ iron tosylate solution (Heraeus), and $3 \mathrm{~g}$ ethanol. The PEG-PDMS-PEG polymer was characterised via ${ }^{1} \mathrm{H}$ NMR spectroscopy (Bruker UltraShield operating at $300 \mathrm{MHz}$ ) and GPC. The results provided a molecular weight $\left(M_{\mathrm{n}}\right)$ of $1800 \mathrm{Da}$ for DBE-U12 and $2000 \mathrm{Da}$ for DBE-U22. The ORR performance of samples based on these two polymers was found to be very similar (Fig. S1 $\dagger$ ) recording values of approximately $-12 \mathrm{~mA} \mathrm{~cm}^{-2}$ at $-0.9 \mathrm{~V}$. Thus for the rest of this study only DBE-U22 has been used.

The carbon paper substrates were supplied from Fuel Cell Earth LLC. PEDOT layers were deposited using vacuum VPP of EDOT monomer (Heraeus) following a method described elsewhere. ${ }^{25}$ In this method the substrates were coated by the oxidant solution via spin coating at $1500 \mathrm{rpm}$ and then exposed to EDOT vapour in a vacuum oven at the pressure of $45 \mathrm{mbar}$ and temperature of $35{ }^{\circ} \mathrm{C}$ for $50 \mathrm{~min}$. The cathodes were then soaked and washed in ethanol to remove any excess oxidant, then dried at $40{ }^{\circ} \mathrm{C}$. The process was repeated so as to form the desired number of layers of PEDOT (i.e. thickness) on the carbon paper.

\section{Electrode testing}

Chronoamperometry was performed to test the oxygen reduction capability of the electrodes using a Voltalab PGZ100, with a Pt wire as the auxiliary electrode and an $\mathrm{Ag} / \mathrm{AgCl}$ reference electrode $(+0.2 \mathrm{~V}$ to NHE). The manufactured electrodes were compared to a commercial, ELAT Pt/C electrode with Pt loading of $0.5 \mathrm{mg} \mathrm{cm} \mathrm{cm}^{-2}$ (NuVant Systems, Inc.). The current density provided by the cathodes was measured at $-300,-500,-700$ and $-900 \mathrm{mV}$, being sustained at the applied voltage for $15 \mathrm{~min}$. Measurements were taken using a $\mathrm{pH} 7$ phosphate buffer solution or $0.1 \mathrm{M} \mathrm{KOH}$. The RDE experiments were performed using PEDOT films deposited on glassy carbon electrodes (RDE, $196 \mathrm{~cm}^{2}$, Pine Research Instrumentation) with a scan rate of 5 $\mathrm{mV} \mathrm{s}^{-1}$. An FEI Quanta 400 microscope was used to obtain SEM images at an accelerating voltage of $20 \mathrm{kV}$.

The efficiency of the three cathodes, when used in batteries, was also tested using an EZstat-pro (NuVant Systems, Inc.). These experiments were carried out using a $4 \mathrm{M} \mathrm{KOH}$ solution as the electrolyte, and utilised a zinc foil (Advent Research Materials, Ltd.) as the anode in the cell structure, as shown in ESI Fig. S2.†

\section{Results and discussion}

\section{Preparation of PEDOT composite electrodes}

Conducting PEDOT films were prepared on substrates (including glass slides and graphite papers) using the vacuum vapour phase polymerisation (VPP) of 3,4-ethylenedioxythiophene (EDOT) as described previously, ${ }^{24,25}$ whereby tosylate anions were used to dope the PEDOT. ${ }^{28}$ Polymeric additives such as PEG-PPG-PEG or PEG-PDMS-PEG were added to the oxidant solution used in the VPP process. Table 1 provides the details of the samples prepared and discussed in this work. After running the polymerisation for 1 hour, the samples were washed with ethanol. At this step the reacted and unreacted oxidant, alongside most of the polymeric additive will be washed off the sample. However, our previous works ${ }^{29}$ have shown that some of the polymeric additive will be trapped and included within the PEDOT structure. In order to confirm that the same statement can be made for PEG-PDMS-PEG block copolymers, X-ray photoelectron spectroscopy (XPS) analysis of 


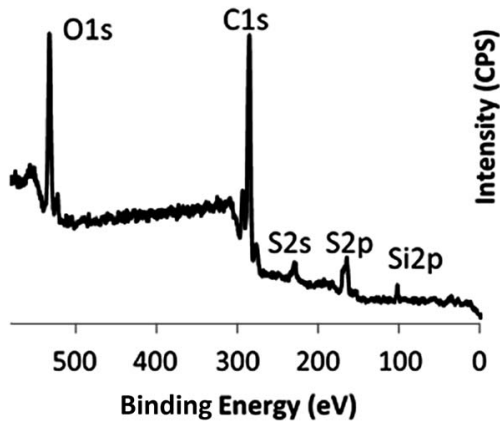

Fig. 1 (a) XPS spectrum of S-PEDOT confirming the presence of silicon-based block copolymer by exhibiting Si2p peak at $101.4 \mathrm{eV}$.

samples was used. XPS results confirmed the presence of the PEG-PDMS-PEG triblock copolymer in the PEDOT film, by exhibiting characteristic Si2p (101.87 eV) peaks (Fig. 1).

It is commonly assumed that greater electrical conductivity is a desirable characteristic for increased ORR efficiency. Measuring the conductivity of a variety of PEDOT composite films based on different polymer additives deposited on glass (Table 1) revealed that the PEDOT composite with the PEG-PDMS-PEG block copolymer (referred to as S-PEDOT) exhibited one of the lowest conductivities $\left(377 \mathrm{~S} \mathrm{~cm}^{-1}\right)$. Blending the PEG-PDMS-PEG copolymer with various amounts of the PEG-PPG-PEG copolymer provided composites with higher conductivities approaching that of PEG-PPG-PEG/ PEDOT composites (referred to as EPE-PEDOT).

SEM images of samples show no significant difference in the morphology of the samples. Fig. S3† shows the SEM images of S-PEDOT and EPE-PEDOT polymers deposited on carbon paper. It can be seen that the porous nature of the substrate is maintained after coating. In agreement with our previous work, ${ }^{20}$ all PEDOT samples form a continuous flat film over the substrate, while the S-PEDOT films appears to be thinner than EPE samples. However, as discussed later, several layers of these films are deposited on the functioning electrodes. By weighting electrodes after deposition of each layer, the mass of each layer of S-PEDOT was measured to be $0.10 \pm 0.03 \mathrm{mg} \mathrm{cm}^{-2}$.

\section{Electrocatalytic activity}

Considering that the electrodes presented in this work are designed to be breathable and absorb oxygen directly from ambient air, only a limited number of electrochemical methods are capable of analysing their performance. Steady-state measurement of conversion current versus applied potential was used to evaluate the performance of the breathable PEDOT composite air-electrodes, as per previous studies. ${ }^{19,20}$ A higher (more negative) current indicates a higher conversion rate and better electrocatalytic efficiency. It should be noted that due to the electroactive nature of PEDOT, conventional voltammetry methods can not differentiate between the current caused by reduction of the polymer and that of the ORR. Therefore, the current density provided by the cathodes was measured after being sustained at the applied potential for 15 min (equilibration time). In this arrangement, considering the small amount of deposited polymer, every redox reaction of the polymer will occur and conclude in the first few seconds, and the reported current values are solely due to ORR.

Maintaining a three-phase interface between the air, the catalytic layer and the electrolyte (Fig. S4†) is critical for efficient electrocatalysis of the ORR., ${ }^{4,20}$ Compared to PEDOT alone, incorporation of PEG in polymer electrodes (E-PEDOT) results in better ORR catalysis by increasing water (as one of the reactants necessary for the ORR) uptake due to the hydrophilicity of PEG. ${ }^{19,20}$ However, the poor absorption and mass transport (diffusion) of oxygen through the electrode has been noted as the main limiting parameter in the ORR cathodes..$^{30,31}$ For this reason, we hypothesised that the incorporation of PEG (a hydrophilic polymer with poor oxygen permeation) is not, in itself, sufficient for achieving good ORR performance. ${ }^{20}$ To produce highly efficient PEDOT-based electrocatalysts for the ORR a key challenge is to develop materials that have catalytic interfaces that are highly accessible to both water and oxygen simultaneously, which has been particularly difficult considering the poor solubility of oxygen in water. In order to test our hypothesis, a range of samples based on different polymer additives were prepared. Table 1 summarises their composition, conductivity and their conversion current value at $-0.9 \mathrm{~V}$ $v s$. $\mathrm{Ag} / \mathrm{AgCl}$ as an indicator of their electrocatalytic activity. It can be seen that incorporation of PEG or PPG does not have a significant effect on the conversion current of electrodes. However, using them beside each other, in form of a block copolymer, can enhance the electrocatalytic activity. Interestingly blending PEG and PPG, in the same composition as the block copolymer, does not result in such improvement. Based on these observation it can be concluded that incorporation of a hydrophobic block that can facilitate oxygen transfer can enhance the electrocatalytic activity. Synthesis of polymers with high oxygen and water absorption has been attracting attention of polymer scientists as a potential material for development of soft contact lenses..$^{32-34}$ A review of the literature in this area reveals that block copolymers of a PDMS and PEG are appropriate materials for achieving such properties. Thus, in the next step PEDOT electrodes based on PEG-PDMS-PEG polymeric additive were prepared. Testing of this sample (S-PEDOT) shows a significantly higher ORR conversion current than any other sample. Interestingly, S-PEDOT demonstrates one of the lowest conductivity values amongst the samples tested in this work, which indicates that simultaneous absorption of oxygen and water has a more significant effect of ORR electrocatalysis than conductivity of electrodes.

Optimisation of the S-PEDOT electrode was first achieved by varying the commercially available carbon supports. Testing electrodes prepared by deposition of S-PEDOT over a wide range of carbon paper substrates (Fig. S5 $\dagger$ ) showed that the 50\% wet-proofed carbon paper can provide the highest conversion current and lowest electrolyte leakage, thus this material was chosen as the preferred substrate.

Our previous work $\mathrm{k}^{\mathbf{2 0}}$ demonstrated that deposition of a thick layer of PEDOT limits the diffusion of oxygen and/or electrolyte through the electrode resulting in a poor three-phase interface, and loss of the ORR performance. In order to determine the 
mass (no. of layers) at which the peak current density occurred, different thicknesses of PEDOT were deposited (1 to 7 layers) (Fig. S6†). The 5-layered sample, corresponding to a mass of $0.5 \mathrm{mg} \mathrm{cm}^{-2}$, exhibited the greatest conversion current at the applied potentials studied. Therefore, for further testing the optimum combination of 5 layers of S-PEDOT on $50 \%$ wet-proofed carbon paper was implemented.

A range of PEDOT composites (Table 1) and ELAT ${ }^{\circledR}$ a commercially available $\mathrm{Pt} / \mathrm{C}$ gas diffusion electrode with a platinum loading of $0.5 \mathrm{mg} \mathrm{cm} \mathrm{cm}^{-2}$ - were compared in terms of their electrocatalytic activity in the ORR. Table 1 lists the steady state conversion current obtained at $-0.9 \mathrm{~V}$, and Fig. $2 \mathrm{a}$ and b demonstrate the conversion current as a function of applied potential in neutral and alkaline buffer solutions, respectively. At a neutral $\mathrm{pH}$ the $\mathrm{Pt} / \mathrm{C}$ electrode produces a higher conversion current than both the PEDOT electrodes at low potentials (Fig. 2a). However, the activity of the S-PEDOT is greatly increased with applied potential, resulting in it outperforming the other samples.

As metal-air batteries and many fuel cell technologies are most commonly operated under alkaline conditions, ${ }^{\mathbf{4}, 30,35}$ the air electrodes were also tested at pH 13 (Fig. 2b). The highest current density achieved under these conditions was $-12.3 \mathrm{~mA}$ $\mathrm{cm}^{-2}$, obtained by the S-PEDOT based air electrode at $-0.9 \mathrm{~V}$, which is significantly higher than that achieved by the other samples. Importantly, such a high conversion current not only surpasses the performance of other PEDOT electrodes examined in this work, but it is also significantly higher than that of the platinum electrodes tested herein (both per area and per weight of catalyst). It is worth-mentioning that in our previous work ${ }^{\mathbf{2 0}}$ where we compared the performance of EPE-PEDOT with a home-made platinum electrode made by sputtering of platinum over a PVDF membrane, a similar performance for EPE-PEDOT and platinum was observed. This is in agreement with the results reported here, based on using a commercial platinum electrode.

In an attempt to improve the performance of the electrodes, PEDOT based on a mixture of PEGPDMS-PEG and PEG-PPG-PEG block copolymers have been prepared. Interestingly, despite improvements in the PEDOT electrode conductivity with the addition of the PEG-PPG-PEG copolymer to composites based on PEG-PDMS-PEG, no additional improvement in the ORR

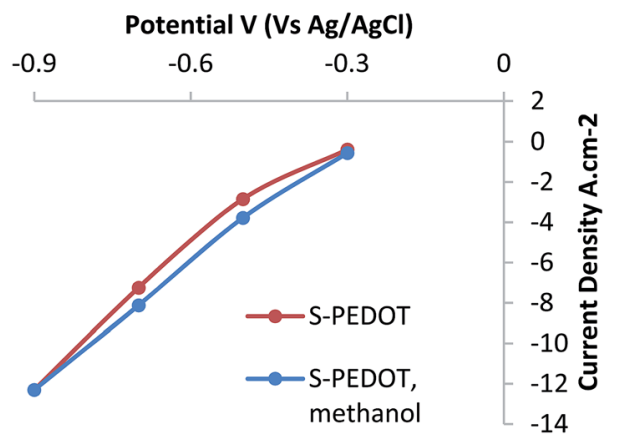

Fig. 3 Steady state conversion current density as a function of applied potential for: pristine S-PEDOT sample and the same sample after soaking in methanol for $20 \mathrm{~h}$.

performance was recorded (Table 1). This indicates that achieving the right balance between oxygen and water absorption on PEDOT electrodes play a more important role than the conductivity of the electrodes.

It is known that methanol poisons Pt based catalysts, ${ }^{19}$ to test the susceptibility of our catalyst to methanol (a possible fuel for fuel cells) the S-PEDOT film was soaked in methanol overnight. Determination of the ORR performance before and after soaking in methanol revealed negligible change in the electrocatalytic performance (Fig. 3). Hence, from this experiment it can be concluded that the polymer catalysts developed in this work are not susceptible to poisoning by methanol.

ORR can occur via two pathways, the 2 electron pathway where oxygen is initially reduced to $\mathrm{H}_{2} \mathrm{O}_{2}$ or $\mathrm{HO}_{2}{ }^{-}$and then reduced to $\mathrm{H}_{2} \mathrm{O}$, and the 4 electron pathway where oxygen is reduced to $\mathrm{H}_{2} \mathrm{O}$ or $\mathrm{OH}^{-}$via transfer of 4 electrons. ${ }^{15,21}$ Rotating disk electrode (RDE) voltammetry methods and development of Koutecky-Levich ${ }^{36}$ plots is a common method for analysis of the ORR mechanism. In this method the number of electrons involved in ORR are estimated by using the slopes of Koutecky-Levich plots (eqn (1)),

$$
\frac{1}{j_{\mathrm{D}}}=\frac{1}{j_{\mathrm{K}}}+\frac{1}{B \omega^{0.5}}
$$

where $j_{\mathrm{K}}$ is the kinetic current density in amperes at a constant potential, $j_{\mathrm{D}}$ is the measured current density on RDE, $\omega$ is the

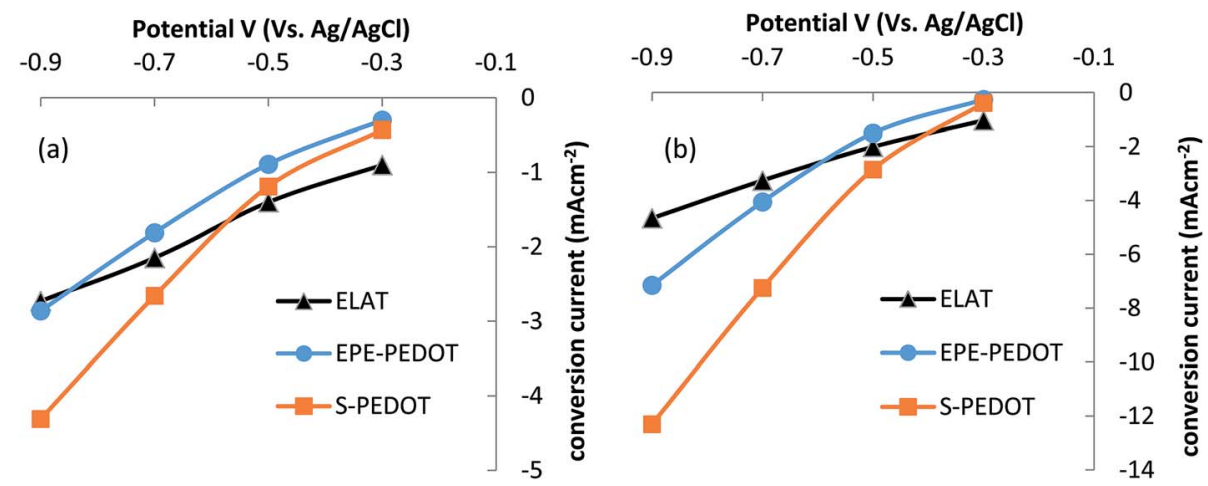

Fig. 2 Steady state conversion current against potential for the ELAT (platinum coated carbon paper), EPE-PEDOT and S-PEDOT electrodes tested at (a) $\mathrm{pH} 7$ and (b) $\mathrm{pH} 13$. 


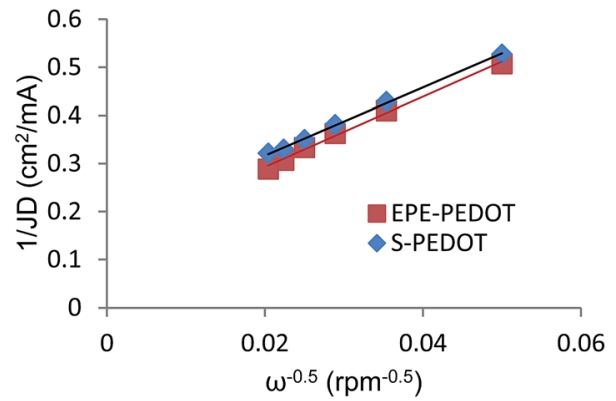

Fig. 4 Koutecky-Levich plots for S-PEDOT and EPE-PEDOT at $-0.5 \mathrm{~V}$ (vs. Ag/AgCl) based on the data presented in Fig. S5 \& S6.† The similar slope observed for both polymeric electrodes indicates a similar ORR mechanism occurring at both electrodes.

electrode rotating speed in rpm, and $B$ is the reciprocal of the slope, which could be determined from the slope of KouteckyLevich plot using Levich equation (eqn (2)):

$$
B=0.2 n F v^{-1 / 6} C D^{2 / 3}
$$

where $n$ is the number of electrons transferred per oxygen molecule, $F$ is the Faraday constant, $v$ is the kinetic viscosity, $C$ is the bulk concentration of $\mathrm{O}_{2}$, and $D$ is the diffusion coefficient of $\mathrm{O}_{2}$ in $0.1 \mathrm{M} \mathrm{KOH}$. The constant 0.2 is adopted when the rotating speed is expressed in rpm.

Due to the physical structure of breathable electrodes, it is not possible to directly evaluate their electrochemical performance using such common methods. However in order to gain insight into the mechanism of the ORR at the PEDOT electrodes, a flat and polished glassy carbon RDE electrodes were coated with a thin layer of PEDOT polymers and their ORR performance in a RDE setup has been studied (Fig. S7 and S8 $\dagger$ ). The overall electron transfer numbers per oxygen molecule involved in the ORR process were calculated from the slopes of Koutecky-Levich ${ }^{36}$ plots (Fig. 4). The results of these experiments reveal a transfer of 3.9 and 4.0 electrons per oxygen molecule for EPE-PEDOT and S-PEDOT electrodes, respectively. So it can be reasonably concluded that both electrodes catalyse the ORR through the 4 electron pathway, and this is in agreement with previous studies on the electrocatalytic behaviour of PEDOT prepared via VPP. ${ }^{21}$ LSV plots at $1600 \mathrm{rpm}$ where also used for estimation of overpotential of the PEDOT electrodes (Fig. S9†). Both EPE-PEDOT and S-PEDOT electrocatalysts show comparable ORR onset potential and low overpotential of 458 and $410 \mathrm{mV}$ versus the equilibrium potential for $\mathrm{O}_{2} / \mathrm{H}_{2} \mathrm{O}$ system at $0.1 \mathrm{M} \mathrm{KOH}$ solution ( $1.23 \mathrm{~V} v$ s. RHE), respectively. Lower overpotential S-PEDOT can be justified by our hypothesis that better access to reactants (i.e. oxygen and water) are resulting in a lower concentration overpotential. Thus, the similar observed ORR pathway and the lower overpotential of S-PEDOT indicate a similar electrocatalysis mechanism for the EPE- and S-PEDOT electrodes while better absorption and diffusion of oxygen is the key contributor towards improved electrocatalytic performance of S-PEDOT.

Besides the desired high ORR efficiency, electrode performance stability over extended periods of time is an essential criterion for developing commercially viable ORR electrodes. The stability of the air-electrodes was studied by measuring their performance over a period of $20 \mathrm{~h}$ (Fig. S10†), which confirmed stable ORR performance for both $\mathrm{S}$ - and EPE-PEDOT composite electrodes. As expected, amongst the two samples tested, S-PEDOT showed the higher average current density.

\section{Zn-air batteries}

To evaluate and compare the performance of the PEDOT composite air-electrodes in a functional battery, zinc-air batteries were prepared by pairing these electrodes with a zinc foil anode in $4 \mathrm{M} \mathrm{KOH}$ solution (Fig. S3†). The open circuit voltages of batteries based on E-PEDOT (a composite based on PEDOT and a PEG homopolymer), EPE-PEDOT and S-PEDOT electrodes were determined to be $1.36,1.42$ and $1.48 \mathrm{~V}$, respectively. The battery based on S-PEDOT exhibited a peak power density of $41.3 \mathrm{~mW} \mathrm{~cm} \mathrm{~cm}^{-2}$ at $0.52 \mathrm{~V}$ (Fig. 5), while the peak power density was 18.9 and $9.1 \mathrm{~mW} \mathrm{~cm}{ }^{-2}$ for batteries based EPE-PEDOT and E-PEDOT cathodes, respectively. It can be seen that changing the polymer additive to PEG-PDMS-PEG resulted in a significant improvement in the peak power density and the voltage of the peak power density. The overall trend observed in the performance of batteries indicate that using additive polymers containing hydrophobic blocks such as PPG and PDMS can effectively improve the performances of the metal-air batteries.
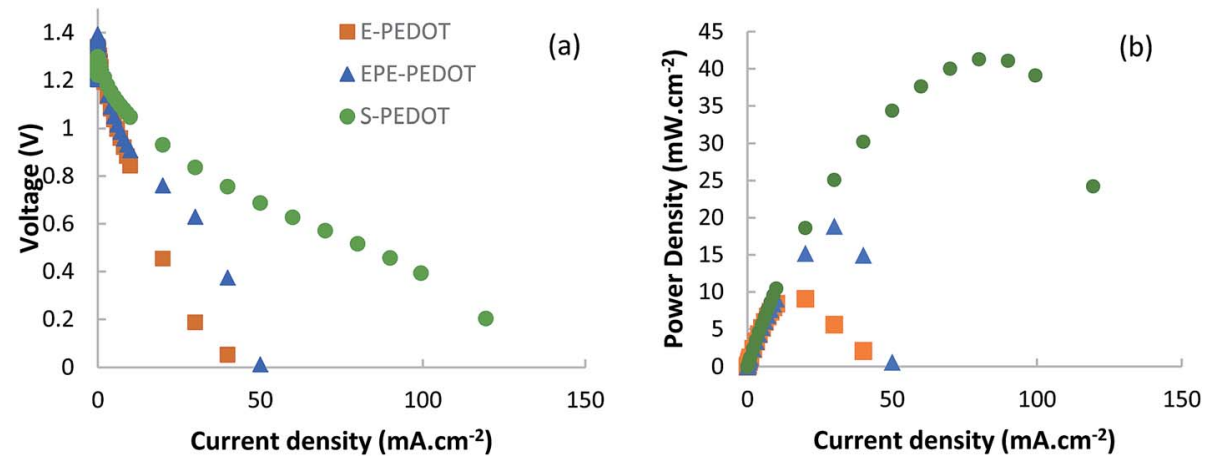

Fig. 5 (a) Polarisation curves and (b) the corresponding power density plots for zinc-air batteries based on polymeric air electrodes. 


\section{Conclusion}

An air-electrode material based on a novel PEDOT composite with the PEG-PDMS-PEG triblock copolymer (referred to as $\mathrm{S}$-PEDOT) has been produced. This cathode showed greater electrocatalytic activity than any other PEDOT electrodes manufactured using other polymeric additives (such as PEG, PPG and PEG-PPG-PEG), and a commercial Pt/C electrode. In comparison to the commercial $\mathrm{Pt} / \mathrm{C}$ electrode, the S-PEDOT electrode has significantly increased the electrocatalytic activity in both neutral and alkaline pH. The S-PEDOT electrodes showed stable ORR performance, and no susceptibility to methanol poisoning, unlike traditional Pt electrodes. The RDE experiments confirmed the proposed mechanism for improved performance of ORR electrodes. The ORR electrodes developed in this work were successfully employed in primary Zn-air batteries with the S-PEDOT electrode showing the highest open circuit voltage $(1.46 \mathrm{~V})$, peak power density $\left(41.3 \mathrm{~mW} \mathrm{~cm}^{-2}\right)$ and energy density ( $715 \mathrm{~W} \mathrm{~h} \mathrm{~kg}^{-1}$ ). The trends observed in performance of batteries were in agreement with other electrochemical assessment results and proposed hypothesis.

\section{Acknowledgements}

P. T. would like to thank the Government of South Australia for the Catalyst Research Grant that was used for funding this project.

\section{References}

1 A. S. Arico, P. Bruce, B. Scrosati, J.-M. Tarascon and W. van Schalkwijk, Nat. Mater., 2005, 4, 366-377.

2 M. Armand and J. M. Tarascon, Nature, 2008, 451, 652-657. 3 J.-S. Lee, S. Tai Kim, R. Cao, N.-S. Choi, M. Liu, K. T. Lee and J. Cho, Adv. Energy Mater., 2011, 1, 34-50.

4 L. Yanguang, G. Ming, L. Yongye, F. Ju, K. Ji-Eun, W. Hailiang, H. Guosong, Z. Bo and D. Hongjie, Nat. Commun., 2013, 4, 1805-1805.

5 Y. Chen, J. Wang, H. Liu, R. Li, X. Sun, S. Ye and S. Knights, Electrochem. Commun., 2009, 11, 2071-2076.

6 S.-Y. Huang, P. Ganesan and B. N. Popov, Appl. Catal., B, 2009, 93, 75-81.

7 C. Wang, N. M. Markovic and V. R. Stamenkovic, ACS Catal., 2012, 2, 891-898.

8 D. He, K. Cheng, H. Li, T. Peng, F. Xu, S. Mu and M. Pan, Langmuir, 2012, 28, 3979-3986.

9 V. Neburchilov, H. Wang, J. J. Martin and W. Qu, J. Power Sources, 2010, 195, 1271-1291.

10 D. Šepa, M. Vojnovíc and A. Damjanovic, Electrochim. Acta, 1970, 15, 1355-1366.

11 A. C. C. Tseung and S. Jasem, Electrochim. Acta, 1977, 22, 3134.

12 J. W. D. Ng, M. Tang and T. F. Jaramillo, Energy Environ. Sci., 2014, 7, 2017-2024.

13 Y. Liang, Y. Li, H. Wang, J. Zhou, J. Wang, T. Regier and H. Dai, Nat. Mater., 2011, 10, 780-786.
14 H. T. Chung, J. H. Won and P. Zelenay, Nat. Commun., 2013, 4, 1922.

15 J. Liang, Y. Zheng, J. Chen, J. Liu, D. Hulicova-Jurcakova, M. Jaroniec and S. Z. Qiao, Angew. Chem., Int. Ed., 2012, 51, 3892-3896.

16 N. Vucaj, M. D. J. Quinn, C. Baechler, S. M. Notley, P. Cottis, P. Hojati-Talemi, M. V. Fabretto, G. G. Wallace, P. J. Murphy and D. R. Evans, Chem. Mater., 2014, 26, 4207-4213.

17 V. G. Khomenko, V. Z. Barsukov and A. S. Katashinskii, Electrochim. Acta, 2005, 50, 1675-1683.

18 B. Kolodziejczyk, O. Winther-Jensen, D. R. MacFarlane and B. Winther-Jensen, J. Mater. Chem., 2012, 22, 10821-10826.

19 B. Winther-Jensen, O. Winther-Jensen, M. Forsyth and D. R. MacFarlane, Science, 2008, 321, 671-674.

20 P. P. Cottis, D. Evans, M. Fabretto, S. Pering, P. Murphy and P. Hojati-Talemi, RSC Adv., 2014, 4, 9819-9824.

21 R. Kerr, C. Pozo-Gonzalo, M. Forsyth and B. Winther-Jensen, ECS Electrochem. Lett., 2013, 2, F29-F31.

22 E. Nasybulin, W. Xu, M. H. Engelhard, X. S. Li, M. Gu, D. Hu and J.-G. Zhang, Electrochem. Commun., 2013, 29, 63-66.

23 D. O. Kim, P.-C. Lee, S.-J. Kang, K. Jang, J.-H. Lee, M. H. Cho and J.-D. Nam, Thin Solid Films, 2009, 517, 4156-4160.

24 P. Hojati-Talemi, C. Bächler, M. Fabretto, P. Murphy and D. Evans, ACS Appl. Mater. Interfaces, 2013, 5, 11654-11660.

25 M. Mueller, M. Fabretto, D. Evans, P. Hojati-Talemi, C. Gruber and P. Murphy, Polymer, 2012, 53, 2146-2151.

26 P. Talemi, M. Delaigue, P. Murphy and M. Fabretto, ACS Appl. Mater. Interfaces, 2015, 7, 8465-8471.

27 C. Gu, B. C. Norris, F.-R. F. Fan, C. W. Bielawski and A. J. Bard, ACS Catal., 2012, 2, 746-750.

28 O. Bubnova, Z. U. Khan, H. Wang, S. Braun, D. R. Evans, M. Fabretto, P. Hojati-Talemi, D. Dagnelund, J.-B. Arlin, Y. H. Geerts, S. Desbief, D. W. Breiby, J. W. Andreasen, R. Lazzaroni, W. M. Chen, I. Zozoulenko, M. Fahlman, P. J. Murphy, M. Berggren and X. Crispin, Nat. Mater., 2014, 13, 190-194.

29 M. V. Fabretto, D. R. Evans, M. Mueller, K. Zuber, P. HojatiTalemi, R. D. Short, G. G. Wallace and P. J. Murphy, Chem. Mater., 2012, 24, 3998-4003.

30 P. A. Christensen, A. Hamnett and D. Linares-Moya, Phys. Chem. Chem. Phys., 2011, 13, 5206-5214.

31 G. U. Sumanasekera, G. Chen, K. Takai, J. Joly, N. Kobayashi, T. Enoki and P. C. Eklund, J. Phys.: Condens. Matter, 2010, 22, 334208.

32 Y.-C. Lai, J. Appl. Polym. Sci., 1995, 56, 317-324.

33 Y. Liu, M. Tu, J. Zhang, Z. Song and Y. Tang, Microporous Mesoporous Mater., 2011, 145, 182-187.

34 J. Yang, G. Wegner and R. Koningsveld, Colloid Polym. Sci., 1992, 270, 1080-1084.

35 Y. Li, W. Zhou, H. Wang, L. Xie, Y. Liang, F. Wei, J.-C. Idrobo, S. J. Pennycook and H. Dai, Nat. Nanotechnol., 2012, 7, 394400.

36 A. J. Bard and L. R. Faulkner, Electrochemical Methods: Fundamentals and Applications, Wiley, New York, 2000. 